American Journal of Applied Sciences 4 (3): 171-175, 2007

ISSN 1546-9239

(C) 2007 Science Publications

\title{
Simplification of Sun Tracking Mode to Gain High Concentration Solar Energy
}

\author{
Omar Aliman, Ismail Daut, Muzamir Isa, Mohd Rafi Adzman \\ School of Electrical System Engineering, Kolej Universiti Kejuruteraan Utara Malaysia (KUKUM) \\ 02600 Jejawi, Perlis, Malaysia
}

\begin{abstract}
Power conversion from solar thermal energy to electrical energy is still very cost-intensive. Serious effort has to be given in the development of the concentrator or heliostat structure expenditure which contributing the most expensive component in a central receiver solar power plant. With current development to find alternatives and lower down the capital, a new mode of sun tracking has been developed and feasibility tested. As it applies a single stage collector replacing conventional double stages structure, the new technique has significantly benefits use in high temperature and high concentration solar energy applications. Meanwhile, the stationary or fixed target (receiver) offers more convenient working environment for various applications. Large and heavy solar powered Stirling Engine could be placed at the stationary location. On the other advantage offers by the new technique, the optical alignment was reasonably easier and less time consuming.
\end{abstract}

Key words: Sun tracking, heliostat, high concentration, solar energy.

\section{INTRODUCTION}

Fixed target type of solar collector such as central power tower ${ }^{[1-4]}$ is usually involves the use of multiple numbers of sun tracking as concentrator devices for bigger scale and higher power comparing to that of moving target solar collectors. The sun tracking which is with plane reflective surfaces however, the targeted images have relative large dimension and limiting the concentration ${ }^{[5]}$. Therefore, it cannot be directly used to apply to the small receiving area of some thermomechanical convector, e.g., the heat part of Stirling engine. Solar furnace on the other hand employing twostage reflection through plane sun tracking and huge parabolic mirror can produce very high concentration to focus the solar rays at small area ${ }^{[6-8]}$. However, this method has to involve high cost and long engineering time, particularly for the precise optical alignments. Therefore, this new technique is proposed to replace the conventional azimuth-elevation tracking mode ${ }^{[9-12]}$ to rotation-elevation tracking mode ${ }^{[13]}$ and concurrently modify the slave mirrors, which are able to be controlled to realize a variable focusing. The working principle of the sun tracking will be illustrated by two parts: primary tracking of heliostat and secondary tracking of slave mirrors.

The principle: In this technique, mirrors are arranged in rows and columns. A master mirror is fixed at the centre. Slave mirrors which share the same frame but have two extra moving freedoms about their pivot points are surrounding the master mirror. Each slave mirror is angularly moved about its pivot point to reflect sun rays into the same fixed target of the master. The result at the target is the total combination of individual mirror images.

Primary tracking: The purpose of primary tracking is to target the sun image of master mirror into a stationary target. Then, this master mirror image acts as a reference for secondary tracking where all the slave mirror images will be projected on it. In Fig. 1, we define $\overrightarrow{\mathrm{ON}}$ as the normal vector of the heliostat front surface; $\overrightarrow{\mathrm{OS}}$ as the vector that points to the sun; $\overrightarrow{\mathrm{OT}}$ as the vector that points to a fixed target. Figure 2 shows the rotation of the plane that contains the three vectors $(\overrightarrow{\mathrm{OS}}, \overrightarrow{\mathrm{ON}}$ and $\overrightarrow{\mathrm{OT}})$ during primary tracking. The tracking movement can be studied by two independent components (Fig. 3). 
a. Rotation movement: The heliostat has to rotate about the TT' axis so that the plane can follow the rotation of the vector $\overrightarrow{\mathrm{OS}}$. As the sun moves through the sky from the morning to solar noon, the plane will rotate starting from horizontal and turning to vertical. The angular movement about this rotation axis is denoted as $\rho$. In Fig. 1, the rotation modes of the sun tracking, $\overline{O N}$ is defined as the normal vector of the heliostat surface; $\overline{O S}$ is the vector that points to the sun;

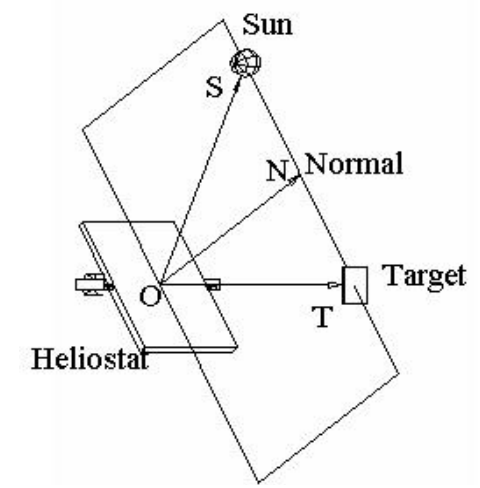

Fig. 1: Rotation modes of sun tracking

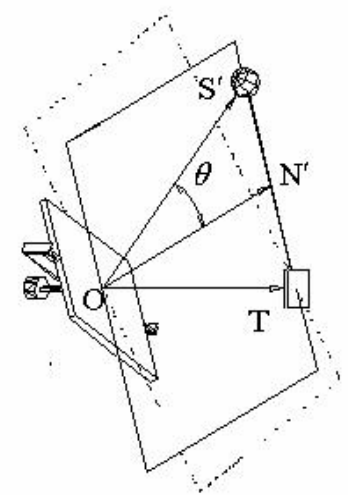

Fig. 2: Rotation plane of sun tracking rotation

$\overline{O T}$ is the vector that points to a fixed target. In Fig. 2, the rotation of the plane of reflection, that which contains the three vectors $(\overline{O S}, \overline{O N}$ and $\overline{O T})$, during primary tracking. In the new reflection plane, the vector $\overline{O S}$ points to the new position of the sun and the vector $\overline{O N}$ ' is the reflector normal of the new orientation so that the sunlight is still reflected towards the target.

Figure 3 illustrates the mounting of sun tracking. which has two tracking axes that are perpendicular to each other, as does the conventional mount. First rotational axis is pointing toward the target and it is indicated by TT' axis. The second axis, elevation axis is attached parallel to the reflector and it is shown as FF' axis.
The coordinate system attached to earth reference frame is illustrated in Fig. 4. The vector $\overline{C S}$ that points towards the sun can be described in terms of hour angle $\omega$ and the declination angle $\delta$. The observer $\mathrm{Q}$ is located at the latitude $\Phi$. By defining a coordinate system with the origin, C, set at the centre of the earth, the $\mathrm{CM}$ axis is a line from the origin to the intersection point between the equator and the meridian of the observer at Q. The CE (east) axis in the equatorial plane is perpendicular to $\mathrm{CM}$ axis. The third orthogonal axis, $\mathrm{CP}$ is the rotation axis of the earth.

The coordinate system attached to the local heliostat reference frame is illustrated in Fig. 5. The vector $\overline{O S}$ is in the function of the angle $\beta$ and the

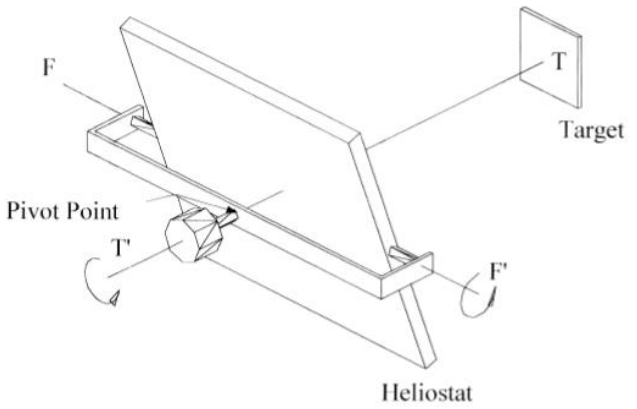

Fig. 3: Sun tracking mounting

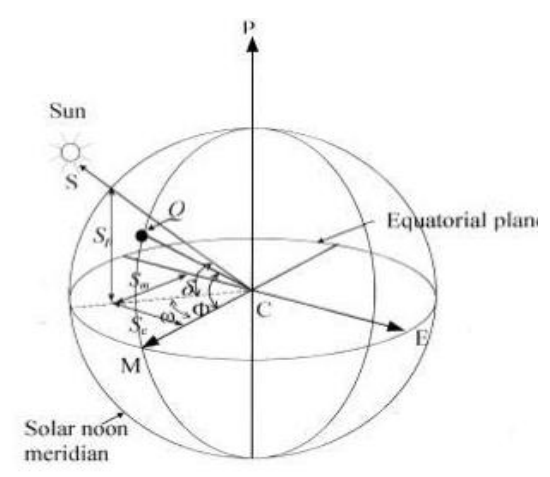

Fig. 4: Coordinate system attached to earth reference frame

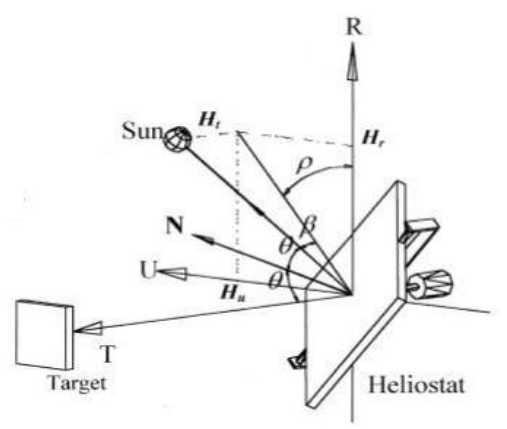

Fig. 5: Coordinate system attached to the local heliostat reference frame 
angle $\rho$. The relationship between the incidence angle $\theta$ and the angle $\beta$ is $\pi / 2=2 \theta+\beta$. The local heliostat reference frame is referred to only when the heliostat frame is oriented in such a way that the normal of master mirror becomes parallel with the rotation axis. The origin of the coordinate system is defined at the centre of the master mirror and is denoted as $\mathrm{O}$. The OR axis is parallel with the array of mirrors arranged in the vertical direction of the heliostat frame. The second axis, OU axis is parallel with the array of mirrors arranged in the horizontal direction. The third orthogonal axis, OT axis is a line pointing out from the origin towards the target direction.

b. Elevation movement: The rotation of the heliostat about FF' axis (perpendicular to the plane) will adjust the heliostat normal position within the plane until it bisects the angle between $\overrightarrow{\mathrm{OS}}$ and $\overrightarrow{\mathrm{OT}}$. As a result, the sunlight will be reflected onto the target. This angular movement depends on the incident angle of the sun relative to the heliostat normal and it is denoted as $\theta$.

The formulas for $\rho$ and $\theta$ can be derived by transformation study of two different coordinate systems: one attaches to the centre of the earth and the other attaches to the heliostat. In Fig. 4, by defining a coordinate system with the origin, $\mathrm{C}$, set at the centre of the earth, the CM axis is a line from the origin to the intersection point between the equator and the meridian of the observer at Q. The CE (east) axis in the equatorial plane is perpendicular to $\mathrm{CM}$ axis. The third orthogonal axis, CP is the rotation axis of the earth. Vector $\overrightarrow{\mathrm{CS}}$ pointing to the sun can be described in terms of its direction cosines, $\mathrm{S}_{\mathrm{m}}, \mathrm{S}_{\mathrm{e}}$ and $\mathrm{S}_{\mathrm{p}}$ to the $\mathrm{CM}$, $\mathrm{CE}$ and $\mathrm{CP}$ axes respectively. Given the direction cosines of $\overrightarrow{\mathrm{CS}}$ in terms of declination angle $(\delta)$ and hour angle $(\omega)$, we have a set of coordinates in matrix form

$\mathrm{S}=\left[\begin{array}{l}\mathrm{S}_{\mathrm{m}} \\ \mathrm{S}_{\mathrm{e}} \\ \mathrm{S}_{\mathrm{p}}\end{array}\right]=\left[\begin{array}{l}\operatorname{Cos} \delta \operatorname{Cos} \omega \\ -\operatorname{Cos} \delta \operatorname{Sin} \omega \\ \operatorname{Sin} \delta\end{array}\right]$

Figure 5 illustrates another coordinate system attached to the heliostat. Similar to the case of $\overrightarrow{\mathrm{CS}}$, vector $\overrightarrow{\mathrm{OS}}$ pointing to the sun can be described in terms of its direction cosines, $\mathrm{H}_{\mathrm{f}}, \mathrm{H}_{\mathrm{r}}$ and $\mathrm{H}_{\mathrm{t}}$ to the $\mathrm{OF}, \mathrm{OR}$ and $\mathrm{OT}$ axes respectively. Given the direction cosines of $\overrightarrow{\mathrm{OS}}$ in terms of $\beta$ and $\rho$, we have a set of coordinates in matrix form
$\mathrm{H}=\left[\begin{array}{l}\mathrm{H}_{\mathrm{f}} \\ \mathrm{H}_{\mathrm{r}} \\ \mathrm{H}_{\mathrm{t}}\end{array}\right]=\left[\begin{array}{l}\operatorname{Cos} \beta \operatorname{Cos} \rho \\ -\operatorname{Cos} \beta \operatorname{Sin} \rho \\ \operatorname{Sin} \beta\end{array}\right]$

where $\beta$ is the angle between the vector $\overrightarrow{\mathrm{OS}}$ and its projection on the surface of heliostat and its relationship with $\theta$ is obviously:

$\theta=\frac{1}{2}\left(\frac{\pi}{2}-\beta\right)$

The new set of coordinate, $\mathrm{H}$, can be interrelated to earth frame based coordinate, S, by three successive rotation transformations.

The first transformation is resulted by a rotation about the CE axis through the latitude angle $\Phi$ (Fig. 4). In matrix notation, it takes the form

$\Phi=\left[\begin{array}{ccc}\operatorname{Cos} \Phi & 0 & \operatorname{Sin} \Phi \\ 0 & 1 & 0 \\ -\operatorname{Sin} \Phi & 0 & \operatorname{Cos} \Phi\end{array}\right]$

The other two transformations take into account of the heliostat initial orientation angles relative to the earth surface, which are facing angle, $\phi$ and target angle, $\lambda$. The facing angle of the heliostat, $\phi$, is the rotation angle about the Zenith made by the heliostat normal when it rotates from the direction towards north to the direction towards a fixed target (assuming that the fixed target and central point of heliostat are at the same horizontal level). Hence, $\phi=0^{\circ}$ if the heliostat is placed due south of the target; $\phi=90^{\circ}$ if the heliostat is located due west of the target. The transformation matrix for the rotation angle $\phi$ about the Zenith is

$\phi=\left[\begin{array}{ccc}1 & 0 & 0 \\ 0 & \operatorname{Cos} \phi & -\operatorname{Sin} \phi \\ 0 & \operatorname{Sin} \phi & \operatorname{Cos} \phi\end{array}\right]$

In general, the central point of heliostat is not at the same horizontal level with the focusing target. Therefore, a rotation transformation through $\lambda$ about OU axis (Fig. 5) is required. $\lambda=0^{\circ}$ means the heliostat's central point is at the same horizontal level as the target; $\lambda=10^{\circ}$ means the OT axis is rotated $10^{\circ}$ clockwise from horizontal line. The transformation matrix is then

$\lambda=\left[\begin{array}{ccc}\operatorname{Cos} \lambda & 0 & \operatorname{Sin} \lambda \\ 0 & 1 & 0 \\ -\operatorname{Sin} \lambda & 0 & \operatorname{Cos} \lambda\end{array}\right]$ 
Finally, $\mathrm{H}$ is the product of $\lambda, \phi, \Phi$ and $\mathrm{S}$ as follows: $\mathrm{H}=\lambda \phi \Phi \mathrm{S}$

From the solutions of the matrix, we obtain $\beta$ and $\rho$ as $\beta=\operatorname{ArcSin}\left\{\begin{array}{l}-\operatorname{Cos} \delta \operatorname{Cos} \omega(\operatorname{Sin} \lambda \operatorname{Cos} \Phi+\operatorname{Cos} \lambda \operatorname{Cos} \phi \operatorname{Sin} \Phi) \\ -\operatorname{Cos} \delta \operatorname{Sin} \omega \operatorname{Cos} \lambda \operatorname{Sin} \phi \\ +\operatorname{Sin} \delta(\operatorname{Cos} \lambda \operatorname{Cos} \phi \operatorname{Cos} \Phi-\operatorname{Sin} \Phi \operatorname{Sin} \lambda)\end{array}\right\}$
$\rho=\operatorname{ArcSin}\left\{\frac{-\operatorname{Cos} \delta \operatorname{Cos} \omega \operatorname{Sin} \phi \operatorname{Sin} \Phi+\operatorname{Cos} \delta \operatorname{Sin} \omega \operatorname{Cos} \phi+\operatorname{Sin} \delta \operatorname{Sin} \phi \operatorname{Cos} \Phi}{\operatorname{Cos} \beta}\right\}(9)$

Eqs. (9) and (3) represent a rotation-elevation tracking mode to perform sun tracking by using a prototype and it has been proven successfully. However, with the movement of the sun tracking of central master mirror, the images of the slave mirrors will be inevitably aberrant. To achieve a high concentration, the slave mirrors need to be adjusted accordingly to overcome this aberration. This adjustment is rather minor, particularly if the target is far away from the heliostat. The principle of this secondary order tracking is illustrated as follow.

Secondary tracking: The new tracking mode encourages the arrangement of the slave mirrors to be grouped into rows and columns as under this mode, the mirrors in the same row or column will have the same movement. Figure 6 shows the side view of a 25-mirror heliostat with $\mathrm{P}$ representing the heliostat frame and the central row (row 3) contains master mirror. Slave mirrors of row 1,2,4 and 5 are attached to the heliostat Sunlight

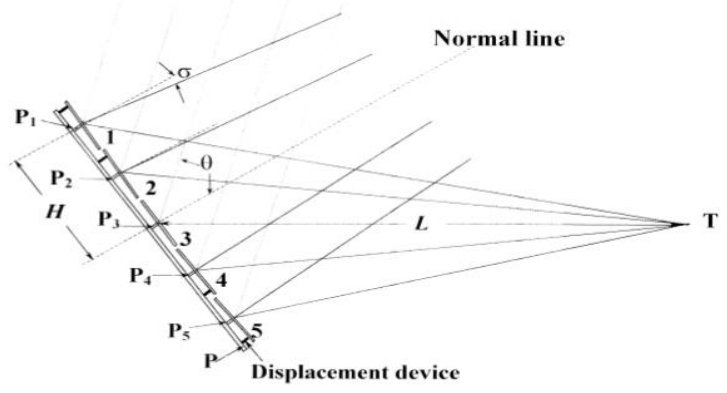

Fig. 6: Side view of a 25-mirror heliostat

frame in such a way that they can turn about their own pivot point $\mathrm{P}_{1}, \mathrm{P}_{2}, \mathrm{P}_{4}$ and $\mathrm{P}_{5}$ respectively. To overlap 4 rows of sun images onto the central master image, each row of slave mirrors has to rotate through an angle, $\sigma$, $\sigma=\frac{1}{2} \operatorname{ArcTan}\left(\frac{\mathrm{HCos} \theta}{\mathrm{HSin} \theta+\mathrm{L}}\right)$

where $\mathrm{L}$ is the horizontal distance between the pivot point of central mirror and the target point Q (Fig. 4); H (positive for rows above the master mirror and negative for rows below the master mirror) is the distance between the centre of master row and the centre of the row where the corresponding slave mirror located.

Similarly, to overlap 4 columns of slave images onto the central mater image, each column has to move through an angle,

$$
\gamma=\frac{1}{2} \operatorname{ArcTan}\left(\frac{\mathrm{H}}{\mathrm{LCos} \theta}\right)
$$

where the definition of $\mathrm{L}$ and $\theta$ remain the same while $\mathrm{H}$ is the distance between the centre of master column and the centre of the column where the corresponding slave mirror located.

The side view of a 25-mirror heliostat with $\mathrm{P}$ representing the heliostat frame is illustrated in Fig. 6. The central row (row 3) contains master mirror. Slave mirrors of row 1, 2, 4 and 5 are attached to the heliostat frame in such a way that they can be turned about their own pivot point $\mathrm{P}_{1}, \mathrm{P}_{2}, \mathrm{P}_{4}$ and $\mathrm{P}_{5}$ respectively.

\section{RESULTS}

To proof the feasibility and reliability of the proposed formulas, tests have been conducted over years since 1997 in Universiti Teknologi Malaysia by two different scales of sun tracking prototypes. A typical structure of sun tracking of this new technique is illustrated in Fig. 7, which has successfully focused images into one fixed target and maintained it throughout the day (Fig. 8).

As an example of its application, a prototype of Sirling engine was tested and successfully operated as illustrated in Fig. 9. The result was excellent where it ran continuously for the whole day without major

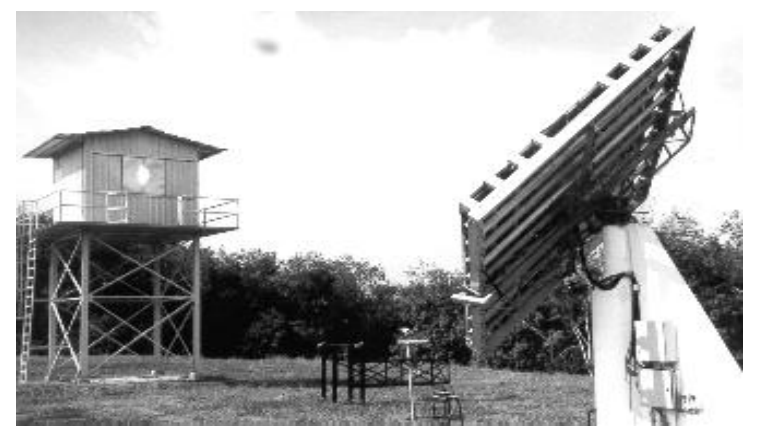

Fig. 7: Prototype of a heliostat focusing to a target 


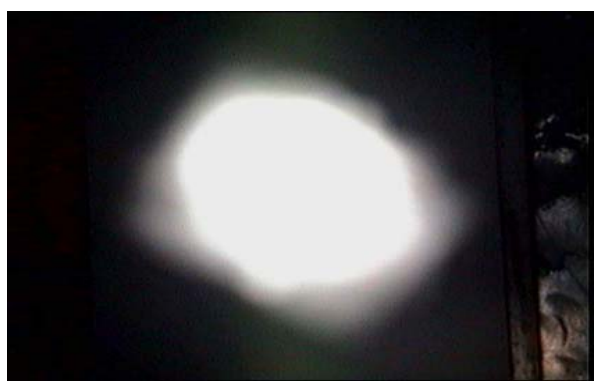

Fig. 8: Daily recorded images

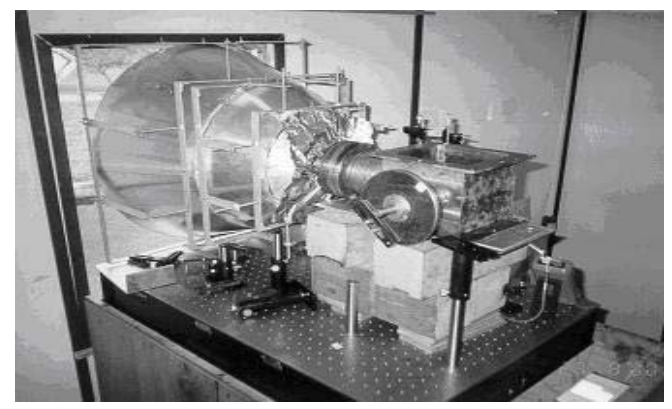

Fig. 9: $\quad$ Stirling engine being tested

problem $^{[15]}$. However, further development to the proposed Stirling engine and other potential applications should be studied seriously to gain the effective result of high concentration from this new technique of sun tracking.

\section{CONCLUSION}

The proposed different technique of sun tracking method has to be explored to make the construction of the sun tracking with many element mirrors cost effective while maintaining a precise sun tracking. In this new technique, by introducing a new rotational axis to the sun tracking frame, the slave mirrors of the same column or the same row can be arranged to share the same driving device. Thus, the number of controlling components can be significantly reduced to $\mathrm{M}+\mathrm{N}$. With this design, the focusing area can be as small as that of an element mirror or even smaller if the element mirrors are concave. Furthermore, different with that of the conventional two-stage solar reflector system, which is rather complicated and costly, in this new technique, the alignment procedure can be simplified, thus, the engineering time can be greatly shortened.

\section{ACKNOWLEDGMENT}

We acknowledge with many thanks to Prof. Y.T.Chen and members of Solar Group which we formerly in the same team and effort, together struggling for the success of this research while we were in University Teknologi Malaysia.

\section{REFERENCES}

1. Takemaro, S. and S. Yukio, 1983. Theoretical concentration of solar radiation by central receiver systems. Intl. J. Solar Energy, 31: 261-270.

2. Bartel, J.J. and P.E. Skvarna, 1984. 10-MWe solar thermal central receiver pilot plant. J. Solar Engg., 106: 50-58.

3. Hirono, T. and T.A. Horigome, 1984. 1-MWe central receiver type solar thermal electric power pilot plant. J. Solar Engg., 106: 90-97.

4. Holmes, J.T., 1982. Heliostat operation at the central receiver test facility, 1978-1980. J. Solar Engg., 104: 133-138.

5. Baranov, V.K., 1980. Heliostats and concentrators with variable reflecting surface geometry. J. Geliotekhnika, 16: 28-33.

6. Azimov, S.A., 1987. Solntse scientific production complex. double-mirror polyheliostat solar furnace with thermal capacity of $1000 \mathrm{~kW}$. J. Geliotekhnika, 23: 3-9.

7. Riskiev, T.T. and K.H. Suleimanov, 1991. Double mirror polyheliostat solar furnace of $1000 \mathrm{~kW}$ thermal power. J. Solar Energy Materials, 24: 625632.

8. Lewandowki, A., B. Carl, O'G. Joseph, W. Roland and S. Dan, 1991. Performance characterization of the seri high-flux solar furnace. J. Solar Energy Materials, 24: 550-563.

9. Alpert, D.J. et al., 1991. Solar concentrator development in the United States. J. Energy Materials, 24: 307-319.

10. Winter, C.-J., R.L. Sizmann and L.L. Vant-Hull, 1991. Solar Power Plants Fundamentals, Technology, Systems, Economics. SpringerVerlag, NY, 1966, pp: 237-250.

11. Fourakis, E. and A.M. Severson, 1977. Low-profile heliostat design for solar central receiver systems. Intl. J. Solar Energy, 19: 349-356.

12. Murphy, L.M., 1986. Stretched-membrane heliostat technology. Intl. J. Solar Energy Engg., 108: 230-238.

13. Chen, Y.T. et al., 2001. Non-imaging focusing heliostat. Intl. J. Solar Energy, 71: 155-164.

14. Chen, Y.T. et al., 2002. Report of the first prototype of non-imaging focusing heliostat and its application in high temperature solar furnace. Intl. J. Solar Energy, 72: 531-544.

15. Tan, K.K. et al., 2000. Solar engine system. Proc. Malaysia Science and Technology Congress, UITM, Shah Alam. 